CONCISE REPORT

\title{
True infliximab resistance in rheumatoid arthritis: a role for lymphotoxin $\alpha$ ?
}

\author{
M H Buch, P G Conaghan, M A Quinn, S J Bingham, D Veale, P Emery
}

Ann Rheum Dis 2004;63:1344-1346. doi: 10.1136/ard.2003.014878

\begin{abstract}
Background: The combination of methotrexate and the antitumour necrosis factor $\alpha(\mathrm{TNF} \alpha)$ antibody infliximab is a very effective treatment for rheumatoid arthritis (RA). However, a proportion of patients are not responsive to this treatment. Inefficacy may represent a TNF $\alpha$ independent disease or insufficient drug at the site of action.

Case report: A patient with RA resistant to repeated high dose infliximab infusions and intra-articular infliximab into an inflamed knee is described. No beneficial clinical effect was observed. Pre-injection arthroscopic biopsy of the study knee demonstrated TNF $\alpha$ staining but also confirmed the presence of lymphotoxin $\alpha$ (LT $\alpha$ or TNF $\beta$ ) on immunohistochemistry. Subsequent treatment with etanercept (which blocks $L T \alpha$ as well as TNF $\alpha$ ) resulted in clinical remission of disease.

Conclusion: This case suggests that resistance to TNF blockade may occur when TNF $\alpha$ is not the dominant inflammatory cytokine and suggests that $\mathrm{LT} \alpha$ may have a pathogenic role in RA.
\end{abstract}

$\mathrm{T}$ he combination of methotrexate and the anti-tumour necrosis factor $\alpha(\mathrm{TNF} \alpha)$ monoclonal antibody infliximab has proved very beneficial in the treatment of rheumatoid arthritis (RA). However, about $35 \%$ of patients do not achieve good clinical improvement. ${ }^{1}$ The reason(s) for this lack of clinical response to $\mathrm{TNF} \alpha$ blockade are unclear. Possible reasons include inadequate levels of drug reaching the primary site of disease or pathophysiological subsets of RA, where the predominant cytokine driving inflammation is not TNF $\alpha$. We report on a patient who failed to respond to both high dose infliximab infusions and intra-articular (IA) infliximab but responded to the alternative TNF $\alpha$ blocking drug etanercept. This case offers new insight into the causes for anti-TNF $\alpha$ non-response.

\section{CASE REPORT}

A 55 year old woman presented with a 4 month history of polyarthritis predominantly affecting hands, wrists, knees, and feet. She had a history of hypothyroidism and was a cigarette smoker with no family history of RA. Her drug treatment was diclofenac $75 \mathrm{mg}$ slow release formulation twice daily, thyroxine $50 \mu \mathrm{g}$ daily, and hormone replacement therapy (oestradiol $2 \mathrm{mg}$ and norethisterone acetate $1 \mathrm{mg}$ ). She had 300 minutes of early morning stiffness (EMS) and on examination had a tender joint count (TJC) of 33 and a swollen joint count (SJC) of 9. Conventional radiography of the hands and feet demonstrated peri-articular soft tissue swelling and osteopenia but no radiographic erosions. Magnetic resonance imaging of her metacarpophalangeal joints confirmed synovitis. She was rheumatoid factor positive, HLA-DR4 positive, had a $\mathrm{C}$ reactive protein (CRP) level of $48 \mathrm{mg} / \mathrm{l}$, and normal thyroid function tests. On the basis of above she was diagnosed as having RA.

After giving informed consent, the patient entered into a research ethics committee approved study employing high dose anti-TNF blockade in patients with early, poor prognosis RA. ${ }^{2}$ In accordance with the study protocol treatment was started with methotrexate $7.5 \mathrm{mg}$ once weekly, folic acid $5 \mathrm{mg}$ twice weekly, and infusions of infliximab $10 \mathrm{mg} / \mathrm{kg}$ at weeks $0,2,6,10$ and then 8 weekly. By week 12 she had no improvement, with EMS 300 minutes, TJC 26, SJC 13, and CRP $41 \mathrm{mg} / \mathrm{l}$. In an attempt to induce significant response the patient underwent a further series of four high dose infliximab infusions at similar time intervals. Over this 6 month period the methotrexate dose was increased from $7.5 \mathrm{mg}$ to $20 \mathrm{mg}$ once weekly. After 6 months of treatment she had EMS 300 minutes, TJC 20, SJC 16, and CRP 125 mg/l. Because she was clearly not responding she was withdrawn from the study.

At withdrawal, her left knee was aspirated and injected with $80 \mathrm{mg}$ of methylprednisolone and she was given $120 \mathrm{mg}$ methylprednisolone intramuscularly. Her left knee had an excellent clinical response to the IA corticosteroid. Despite this she then continued to have very active disease in most of her other joints. As her right knee continued to be painful and swollen she underwent an arthroscopy in March 2000. This demonstrated typical RA findings of moderate synovial membrane villous formation and marked vascularity. Five weeks after this procedure her arthroscopic portals were healed but there was no change in her disease state, including persistent symptoms and signs in her right knee. To examine the TNF $\alpha$ resistance, IA infliximab was given. Previous reports have demonstrated the effectiveness of IA TNF blocking agents both locally and systemically. ${ }^{3}$ The right knee was injected with $70 \mathrm{mg}$ (in $7 \mathrm{ml}$ solution) of infliximab. The patient was reviewed daily over the next 5 days and no adverse event was recorded. However, the patient reported no change in pain in the right knee and there was no change in the effusion present. At day 5 the knee was aspirated and $80 \mathrm{mg}$ of IA methylprednisolone was injected. The knee had an excellent clinical response. With the patient continuing to have very active disease, sulfasalazine and hydroxychloroquine were added to her second line treatment (sulfasalazine dose increased to $1 \mathrm{~g}$ daily over 2 weeks). The following month sulfasalazine was stopped owing to gastrointestinal side effects. Her methotrexate dose was consequently increased to $25 \mathrm{mg}$ once weekly (over 4 weeks).

The patient continued to show an unacceptable level of disease activity. Consequently, hydroxychloroquine was stopped and treatment was started with etanercept subcutaneously $25 \mathrm{mg}$ twice weekly. By week 12 of the

Abbreviations: CRP, $C$ reactive protein; EMS, early morning stiffness; HACAs, human anti-chimeric antibodies; IA, intra-articular; LT $\alpha$, lymphotoxin $\alpha ; R A$, rheumatoid arthritis; SJC, swollen joint count; TJC, tender joint count; $T N F \alpha$, tumour necrosis factor $\alpha$ 


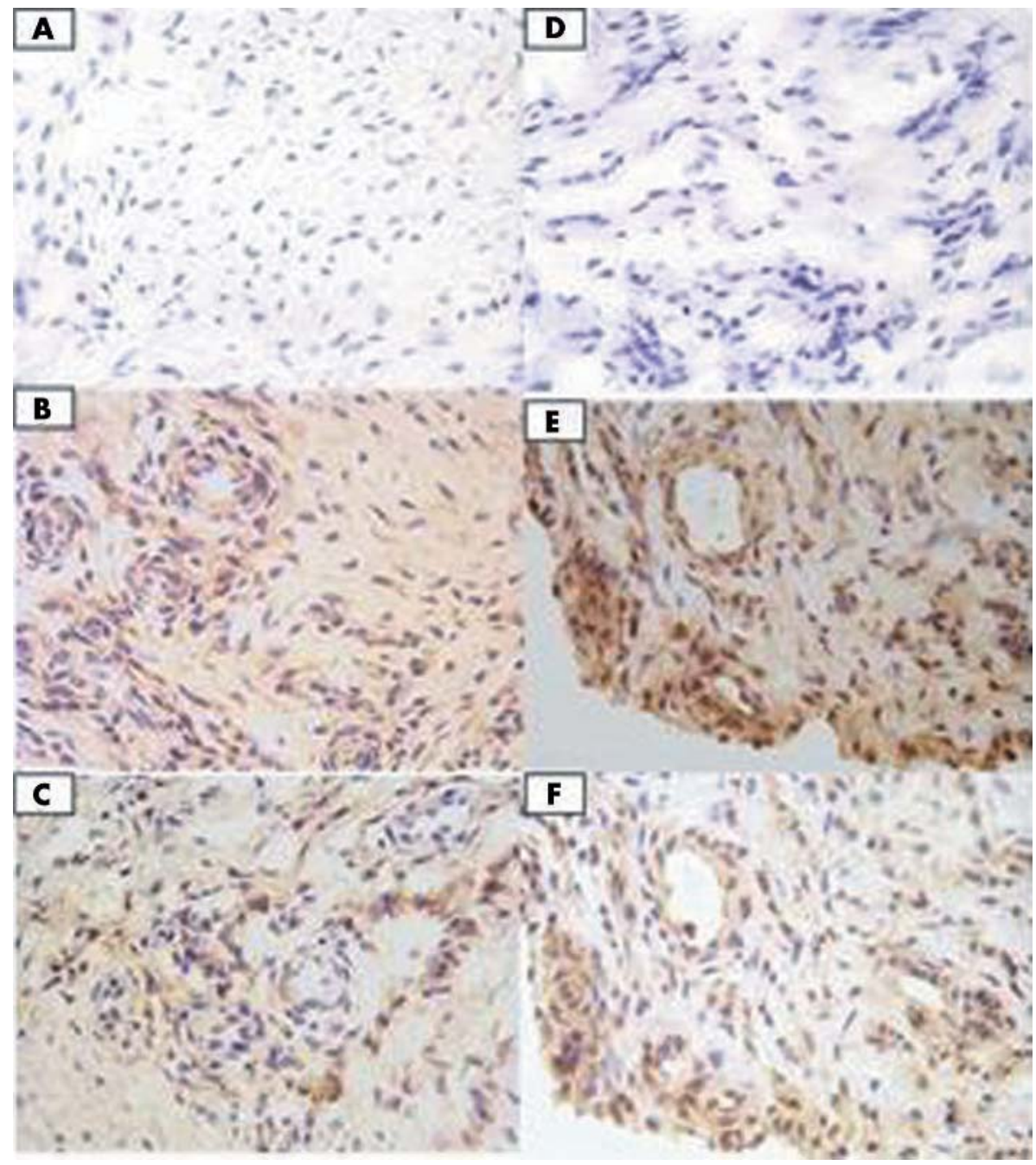

Figure 1 Panels (A) and (D) are the negative controls for the study patient and positive control respectively. Panels (B) and (E) demonstrate TNF $\alpha$ expression detected with anti-TNF $\alpha$ monoclonal mouse lgG1 clone 28401 in the study patient and positive control, respectively. Panels $(C)$ and $(F)$ demonstrate $\mathrm{LT} \alpha$ expression detected with anti-LT $\alpha$ monoclonal mouse $\operatorname{lgG} 1$ clone 5802.21 in the study patient and positive control, respectively.

etanercept treatment a dramatic symptomatic and clinical response was observed. She had no EMS and on examination TJC 2, SJC 1, and normal CRP. "Good" EULAR and ACR50 responses were achieved. By 9 months her ACR response had improved to ACR70. She has continued to be in clinical remission for over $2 \frac{1}{2}$ years.

Synovial membrane biopsy specimens obtained from arthroscopy were embedded at optimum cutting temperature on cold hexane, snap frozen, and stored at $-80^{\circ} \mathrm{C}$. Cryostat sections ( $3 \mu \mathrm{m}$ and $4 \mu \mathrm{m}$ thick) were mounted on superfrost slides (Surgipath) and dried overnight at $37^{\circ} \mathrm{C}$. Sections were fixed for 20 minutes in acetone before incubating with monoclonal antibodies anti-TNF $\alpha$ (monoclonal mouse IgGl clone 28401 R\&D Systems) and anti-lymphotoxin $\alpha$ (LT $\alpha$ ) (monoclonal mouse IgGl clone 5802.21 R\&D Systems) for 1 hour. A standard staining procedure using ChemMate (DAKO) was used. Endogenous peroxidase activity was blocked for 10 minutes. Secondary biotinylated antibody was added and incubated for 30 minutes, followed by addition of horseradish peroxidase conjugated avidin-biotin complex for another 30 minutes. 3,3'-Diaminobenzidine was used to develop colour and was terminated with serial washings at 10-15 minutes. The slides were counterstained in haematoxylin (Sigma), dehydrated in ethanol and xylene, and then mounted.
Arthroscopic inspection disclosed typical RA synovitis, with villous proliferation. Histology confirmed dense cellular infiltration. Expression of TNF $\alpha$ was seen in the lining and sublining layers. Staining for $\mathrm{LT} \alpha$, however, was also positive again in both the lining and sublining layers (fig 1). The positive control used was another patient with RA with no previous exposure to biological agents.

\section{DISCUSSION}

This report presents a patient with recent onset RA who deteriorated while receiving repeated high dose intravenous infusions, flared after IA infliximab but who showed a dramatic response (achieving remission) to etanercept, an alternative TNF $\alpha$ blocking treatment. Infliximab is a chimeric monoclonal antibody composed of the variable region of mouse anti-human TNF $\alpha$ antibody fused to human IgGl. Infliximab binds and neutralises both soluble and membrane-bound TNF $\alpha$ and can lyse cells bearing the latter through complement activation or antibody dependent, cell mediated cytotoxicity. Etanercept is a dimeric TNF receptor:IgGl fusion protein that neutralises both TNF $\alpha$ and (unlike infliximab) LT $\alpha$, but does not possess the cytotoxic effect in vitro. The contrasting effects of etanercept following infliximab raises various questions about the complex issue of non-response. 
A number of factors can be considered, the first being whether the response or non-response is a product of the current scoring tools used. Both the total lack of response with infliximab and the degree of response seen with etanercept were dramatic and at the extreme; the differences could not be explained by an artefact of measurement. Low serum drug levels might also contribute to a diminished response but would explain neither failure of response nor the flare of disease as seen here. Furthermore, the administration of an intravenous, high dose drug with a loading regimen is likely to ensure appropriate serum levels, avoiding potential pharmacokinetic issues. Another reason cited for infliximab non-response is the formation of human antichimeric antibodies (HACAs). Again we do not believe our case represents this primarily for two reasons. Firstly, with HACAs a clinical response is initially seen, which is later lost. As stated above, our patient failed to show any signs of improvement with high dose infliximab at any time including immediately after the first infusion. Secondly, the knee joint was treated with intra-articular infliximab. The infliximab dose given would overwhelm any HACAs present, yet this injection produced a flare of disease. As none of these possible explanations seem likely this suggests that other cytokines within the complex network may possess a pathogenic role. The presence of LT $\alpha$ staining in the preinjection synovial biopsy specimen of our patient may be relevant to explaining the subsequent outcome.

Evidence suggests that chronic inflammation has many of the characteristics of lymphoid organ neogenesis, and that LT $\alpha$ seems to have a crucial role in both. LT $\alpha$ is a member of the TNF family, also known as TNF $\beta{ }^{4}$ It binds to TNFRl (p55) and TNFR2 (p75). Knockout mice models have demonstrated the crucial role of LT $\alpha$ in lymphoid organ development. ${ }^{56} \mathrm{LT} \alpha$, however, also exhibits proinflammatory effects and induces inflammation in in vivo studies of transgenic mice, confirming earlier in vitro studies. ${ }^{78}$

This is the first report of such diametrically opposed responses to infliximab and etanercept. The detection of synovial LT $\alpha$ (as well as TNF $\alpha$ ) expression raises the interesting possibility that LT $\alpha$ has at least a partial role in disease drive and pathogenesis. To add further weight to this concept further synovial studies correlating LT $\alpha$ and TNF $\alpha$ expression with response and non-response to infliximab are required. The case, however, epitomises the phenomenon of true resistance to infliximab, prompting the need for further investigation.

\section{ACKNOWLEDGEMENTS}

We acknowledge the technical assistance provided by Anne English and Karen Henshaw.

We are also grateful to Schering-Plough UK for the supply of infliximab.

\section{Authors' affiliations}

M H Buch, P G Conaghan, M A Quinn, S J Bingham, D Veale, P Emery, Academic Unit of Musculoskeletal Disease, University of Leeds, UK

Correspondence to: Professor P Emery, Academic Unit of Musculoskeletal Disease, 1 st Floor, Leeds General Infirmary, Great George Street, Leeds LS1 3EX, UK; p.emery@leeds.ac.uk

Accepted 25 November 2003

Published online first 19 March 2004

\section{REFERENCES}

1 Maini R, St Clair EW, Breedveld F, Furst D, Kalden J, Weisman M, et al. Infliximab (chimeric anti-tumour necrosis factor alpha monoclonal antibody) versus placebo in rheumatoid arthritis patients receiving concomitant methotrexate: a randomised phase III trial. Lancet 1999;354:1932-9.

2 Conaghan PG, Quinn M, O'Connor P, Wakefield RJ, Karim Z, Emery P. Can very high-dose anti-tumour necrosis factor blockade at onset of rheumatoid arthritis produce long-term remission? Arthritis Rheum 2002;46:1971-3.

3 Dreher R, Flaig W, Leitzke D. Treatment of rheumatoid arthritis by intraarticular injections with TNF-alpha blockers [abstract]. Arthritis Rheum 2001;44(suppl):S42.

4 Paul NL, Ruddle NH. Lymphotoxin. Ann Rev Immunol 1988;6:407.

5 De Togni PD, Goellner J, Ruddle NH, Streeter PR, Fick A, Mariathasan S, et al. Abnormal development of peripheral lymphoid organs in mice deficient in lymphotoxin. Science 1994;264:703-7.

6 Banks TA, Rouse BT, Kerley MK, Blair PJ, Godfrey VL, Kuklin NA, et al. Lymphotoxin-alpha-deficient mice: effects on secondary lymphoid organ development and humoral immune responsiveness. J Immunol 1995; 144:1685-93.

7 Kratz A, Campos-Neto A, Hanson MS, Ruddle NH. Chronic inflammation caused by lymphotoxin is lymphoid neogenesis. J Exp Med 1996;183:1461-72.

8 Sacca R, Cuff CA, Lesslauer W, Ruddle NH. Differential activities of secreted lymphotoxin-3 and membrane lymphotoxin-1 $\beta 2$ in lymphotoxin-induced inflammation: critical role of TNF receptor 1 signaling. J Immunol 1998; 160:485-91. 